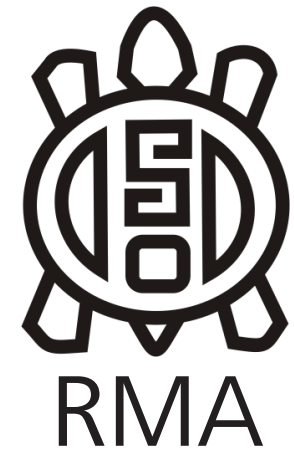

Dossier

\title{
El poblamiento del bosque del centro-norte de la Patagonia argentina: nuevos datos del Parque Nacional Lago Puelo (provincia del Chubut)
}

\author{
The peopling of North-Central Patagonia forest: new data from Lago \\ Puelo National Park (Chubut province, Argentina)
}

Pablo Marcelo Fernández*, Cristina T. Bellelli**, Mariana Carballido Calatayud*, M. Mercedes Podestá***, Soledad Caracotche****, Sabrina Leonardt**, Mercedes G. Fernández** y Ana Forlano***

* CONICET, Instituto Nacional de Antropología y Pensamiento Latinoamericano (Ministerio de Cultura de la Nación), Universidad de Buenos Aires, Argentina. E-mail: pablomfernandez69@yahoo.com.ar, mcarballidocalata@hotmail.com

** CONICET, Instituto Nacional de Antropología y Pensamiento Latinoamericano (Ministerio de Cultura de la Nación), Argentina. E-mail: crisbellelli@yahoo.com.ar, sabrinaleonardt@yahoo.com.ar, fernandez_mercedesgrisel@hotmail.com

*** Instituto Nacional de Antropología y Pensamiento Latinoamericano (Ministerio de Cultura de la Nación), Argentina. E-mail: mercedespodesta@yahoo.com, anaforlano@gmail.com

**** Administración de Parques Nacionales, Dirección Regional Patagonia Norte, Argentina. E-mail: scaracotche@apn.gob.ar

\begin{abstract}
Resumen
La historia de uso del bosque del centro-norte de la Patagonia argentina revela la heterogeneidad de respuestas en la ocupación humana de este ambiente a través del tiempo y el espacio. Con el objetivo de dar cuenta de estas variaciones, nos propusimos evaluar el alcance del modelo de poblamiento propuesto para nuestra área de investigación en el SO de Río Negro - NO de Chubut, en el Parque Nacional Lago Puelo- Parque Provincial Río Turbio. Estas áreas protegidas se caracterizan por una extensa red fluvio-lacustre, pasos cordilleranos de baja altura que conectan con el Pacífico y diferentes configuraciones de bosque en un espacio acotado producto del pronunciado gradiente de precipitaciones. La cronología obtenida remite al final del Holoceno tardío, el estilo de las manifestaciones rupestres es congruente con este lapso y presentan cierto grado de variación local. Predominan las evidencias de circulación terrestre y no se hallaron testimonios de navegación. Los sitios se concentran en un área acotada y la excavación de dos de ellos proporcionó escasa evidencia arqueológica, que indica el uso de recursos locales y de otros biomas. Estos resultados muestran coincidencias con otras zonas del bosque y particularidades cuyo significado deberán precisarse con los trabajos en curso.
\end{abstract}

Palabras clave: Holoceno tardío; Cazadores-recolectores; Contacto; Circulación pedestre y navegación; Arte rupestre.

\begin{abstract}
The occupational history of the Andean Forest from North-Central Patagonia, Argentina, reveals temporal and spatial variations in the human responses to the peopling of this environment. To deepen the knowledge about this variability, we evaluate the population model we proposed for SW Río Negro - NW Chubut forest, at Lago Puelo National Park-Río Turbio Provincial Park. The area is characterized by an extensive fluvial-lacustrine network, low mountain passes that connect to the Pacific coast, and different forest types that are a result of the sharp precipitation gradient. We dated the archaeological sites at the end of the late Holocene, and the rock art style is congruent with this time. It is similar to the paintings recorded in neighbors' areas, but some local variations were identified. Evidence of terrestrial circulation predominates, and no evidence of navigation was collected. The sites are spatially concentrated, and the excavation of two of them provided scant archaeological evidence. However, they indicate the use of local (Hippocamelus bisulcus) and non-local (obsidian, marine shell) resources. These results indicate coincidences with other areas of the forest and some particularities. The research's next steps will aid clarified the meaning of the differences.
\end{abstract}

Keywords: Late Holocene; Hunter-gatherers; European-native interactions; Rock art; Navigation and pedestrian transit. 


\section{Introducción}

Las investigaciones sobre el poblamiento de los bosques mixtos de Nothofagus spp. - Austrocedrus chilensis del centro-norte de la Patagonia argentina revelan una historia de uso humano compleja y variable a través del tiempo (Fernández et al. 2013; Hajduk et al. 2018; Lezcano et al. 2010; Pérez 2010; Pérez y Reyes 2009; Silveira et al. 2013, 2014). Los indicios más antiguos de la presencia humana en estos bosques se remontan al inicio del Holoceno (10600 años AP) en el sitio El Trébol, en ese momento ubicado en un bosque abierto cercano al ecotono entre el bosque y la estepa (Hajduk et al. 2006). En el interior del bosque, estas evidencias se observan unos milenios más tarde en el sitio Población Anticura, cuya ocupación más antigua data de 8200 años AP (Fernández et al. 2019a). Luego, en ambos sitios se registra un lapso considerable sin testimonios, los que reaparecen hace 5800-5600 años AP en El Trébol y hace 4700 años AP en Población Anticura (Fernández et al. 2019a, Hajduk et al. 2006). Después de estas discretas ocupaciones del Holoceno medio hay un nuevo hiato hasta el Holoceno tardío (Fernández et al. 2013, 2019a; Hajduk et al. 2006). Aproximadamente hacia 3500 años AP comienza a incrementarse la señal arqueológica, fenómeno que se percibe a lo largo de los $300 \mathrm{~km}$ de bosque comprendidos entre $40^{\circ} 20^{\prime} \mathrm{S}$ y $43^{\circ}$ 10’ S (Fernández et al. 2013).

El uso discontinuo observado entre las primeras ocupaciones y los 3500 años AP ha dejado escasas evidencias materiales, algunas de la cuales pueden ser vinculadas con otros ambientes, como la estepa o la costa marina (Bellelli et al. 2018, Fernández et al. 2019a). Los conjuntos arqueológicos de este lapso fueron generados por grupos pequeños durante estancias de corta duración, en el marco de un uso logístico del bosque (Fernández et al. 2019a, Lezcano et al. 2010). Entre 3500 y ca. 2000-1700 años AP las visitas al bosque son más asiduas, presumiblemente bajo el mismo régimen logístico. Luego, en el último tramo del Holoceno, se observa un incremento notable en la cantidad de sitios, la mayoría con manifestaciones rupestres (Fernández et al. 2013). Mientras que el aumento en el número de sitios se observa en varias zonas de bosque entre las latitudes mencionadas más arriba, en nuestra área de investigación vemos además una mayor redundancia ocupacional de ciertos sitios, el incremento en la cantidad de materiales depositados, el uso de la cerámica y la presencia de restos humanos cuya señal isotópica indica el consumo de recursos del bosque / ecotono bosqueestepa (Fernández et al. 2013; Fernández y Tessone 2014). Asimismo, registramos la incorporación de roedores Caviomorfos, puma (Puma concolor) y, en tiempos históricos, caballo (Equus caballus) a la subsistencia. Estos recursos se suman al huemul (Hippocamelus bisulcus), que fue la presa principal durante todo el Holoceno, y al pudú (Pudu puda) y al guanaco (Lama guanicoe), que siempre fueron complementarios (Fernández y Fernández
2019). Este conjunto de indicios permite proponer una mayor permanencia de las poblaciones dentro del bosque (Fernández et al. 2013), que habría sido posible a partir de una alta movilidad residencial (Fernández y Carballido Calatayud 2015), el empleo del arco y flecha (Carballido Calatayud y Fernández 2020), el uso generalizado de la cerámica (Bellelli 2020, Bellelli et al. 2020) y el acceso a recursos y/o redes sociales por fuera de este ambiente (Bellelli et al. 2008, 2018; Leonardt 2016). La idea de visitas más asiduas y estancias más prolongadas dentro del bosque podría tener un apoyo adicional en la existencia de una variante de la Tendencia Abstracta Geométrica Compleja (TAGC, Gradin 1999), la Modalidad del Ámbito Lacustre Boscoso (MALB, Albornoz y Cúneo 2000), que ha sido registrada en representaciones rupestres de nuestra área de estudio (Podestá et al. 2020). La ocupación permanente o semipermanente del bosque durante el tramo final del Holoceno se habría dado también en otras áreas del centro-norte de Patagonia (Hajduk et al. 2011; Lezcano et al. 2010; Pérez 2010; Pérez y Reyes 2009; Pérez et al. 2019).

El objetivo de este trabajo es continuar evaluando el alcance del modelo de poblamiento propuesto por nuestro equipo en nuevos sectores del bosque andino del centro-norte de Patagonia, en particular en la zona del Parque Nacional Lago Puelo (PNLP) y el contiguo Parque Provincial y Reserva Forestal de Uso Múltiple Río Turbio (en adelante, PP, Figura 1). Esta zona se caracteriza por su extensa red fluvio-lacustre y por la existencia de pasos de baja altura que permiten acceder al litoral pacífico. Además, el pronunciado gradiente de las precipitaciones, que disminuyen de Oeste a Este de 1500 a 800 mm anuales promedio en tan sólo $15 \mathrm{~km}$, produce diferentes configuraciones de bosque en un espacio acotado (Faggi 1994). Estas particularidades la convierten en una zona atractiva para evaluar el peso de la movilidad lacustre y terrestre, la interacción con otros biomas y el uso de los distintos tipos de bosque. A continuación, desarrollamos una puesta al día de los conocimientos arqueológicos adquiridos en esta área desde 2012, año en que iniciamos las investigaciones sistemáticas y de las que sólo habíamos dado a conocer algunos avances (Bellelli et al. 2014) o aspectos muy específicos (Caracotche et al. 2013; Masotta et al. 2016).

\section{Antecedentes arqueológicos}

Las investigaciones arqueológicas en el PN Lago Puelo se remontan al trabajo pionero de Nicolás Sánchez Albornoz, quien en 1955 documenta tres sitios con manifestaciones rupestres: A Orillas del lago Puelo (OLP), En la subida de la vega del Turbio (SVT) y Recodo del Turbio (RDT), este último ubicado en el actual PP (Sánchez Albornoz 1958). En ocasión de un reconocimiento de estos sitios, Roberto Molinari (Administración de Parques Nacionales, en adelante APN) identifica en 1991 otros dos sitios con pinturas denominados Escuela 186 (ESC186) y 


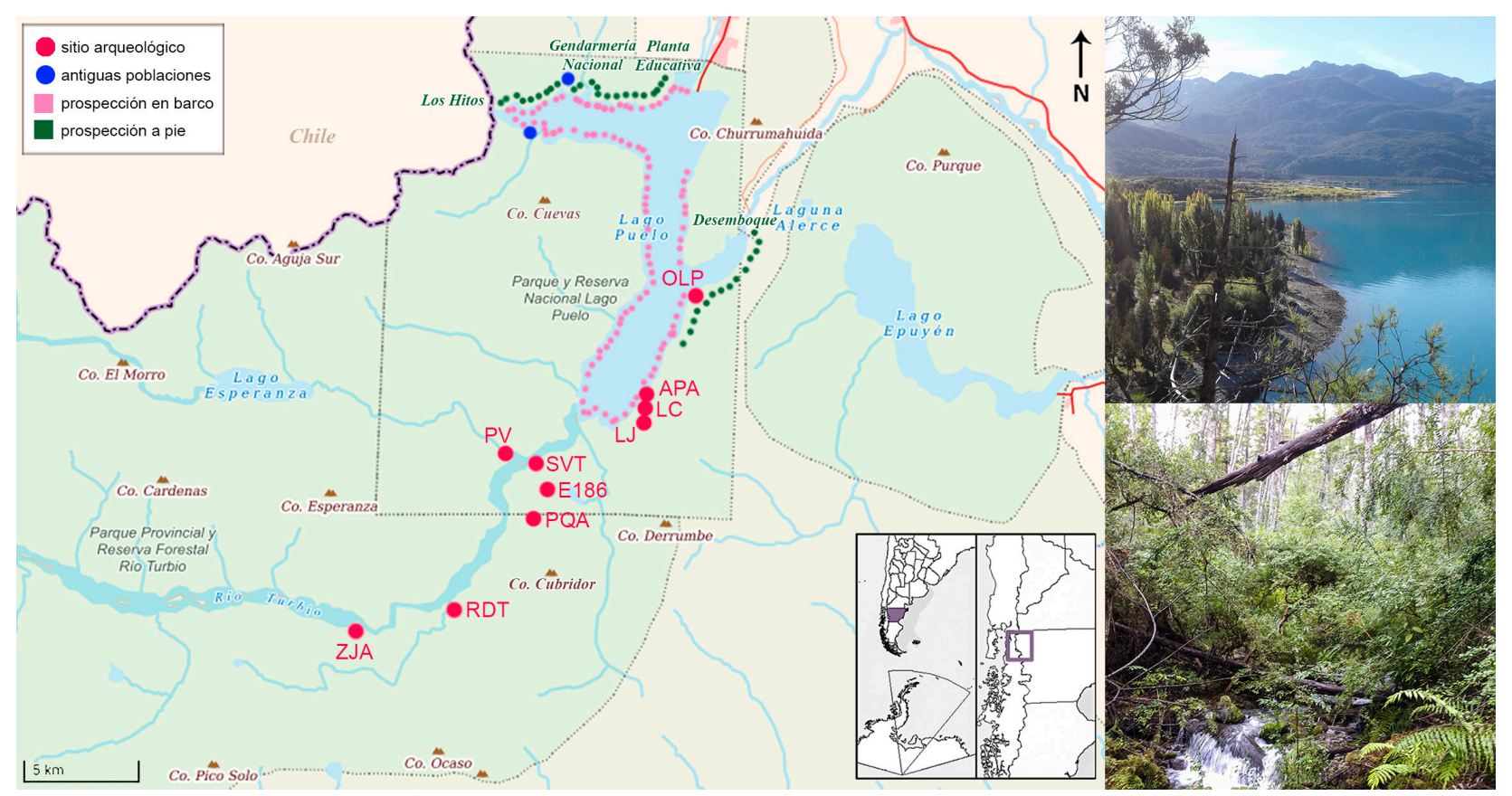

Figura 1. Mapa del Parque Nacional Lago Puelo y Parque Provincial y Reserva Forestal Río Turbio en el que se indican las prospecciones a pie y en embarcación, la ubicación de los sitios arqueológicos y de las "antiguas poblaciones". Mapa base extraído de Argenmap/ Instituto Geográfico Nacional. Referencias de los sitios: en sentido Norte-Sur, OLP, Orillas del Lago Puelo; APA, Alero de la Piedra Alta; LC, Los Círculos; LJ, La Jarra; PV, Piedra Vigueras; SVT, Subida a la Vega del Turbio; E186, Escuela 186; PQA, Puesto que Arde; RDT, Recodo del Turbio y ZJA, Zanjón del Avellanal. Fotos: arriba, vista del lago Puelo y desembocadura de El Turbio; abajo, bosque cerrado en el sector de mayor precipitación del Parque Nacional.

Figure 1. Map of surveys and archaeological sites in the Lago Puelo National Park and Río Turbio Provincial Park and Forest Reserve (Chubut, Argentina). Map source: Argenmap/Instituto Geográfico Nacional. Site references: (North-South oriented) OLP, Orillas del Lago Puelo; APA, Alero de la Piedra Alta; LC, Los Círculos; L, La Jarra; PV, Piedra Vigueras; SVT, Subida a la Vega del Turbio; E186, Escuela 186; PQA, Puesto que Arde; RDT, Recodo del Turbio, and ZJA, Zanjón del Avellanal. Photos: top, Puelo lake and El Turbio river mouth; bottom, closed forest in the highest rainfall section of the Puelo National Park.

Piedra Vigueras (PV, Molinari 1991). Posteriormente, en 1995 se llevó a cabo un nuevo reconocimiento de los sitios con pinturas conocidos y se sumó el sitio Puesto que Arde (PQA, Podestá et al. 2000). Estos trabajos estuvieron orientados a la identificación, documentación y conservación de los sitios con manifestaciones rupestres (Podestá et al. 2000). Asimismo, los informes del personal de la APN representan otro antecedente: uno da cuenta del hallazgo del sitio La Jarra (LJ, Ballejos 1999), otro de una punta de calcedonia recuperada en una playa de la costa Norte del brazo occidental del lago (Ocampo y Giachino 2005) y un tercero informa sobre las pinturas en el sitio Los Círculos (LC, López 2015). A partir de 2012, nuestro equipo inicia una nueva etapa de los trabajos en el área con el desarrollo de prospecciones, relevamientos y excavaciones, que se informan en este trabajo.

\section{Metodología}

\section{Prospecciones}

Las restricciones de visibilidad arqueológica que presenta el ambiente de bosque (Scheinsohn 2004) hacen que la búsqueda de las evidencias materiales dependa, en una primera instancia, de la información de los pobladores. Esta fuente es la que utilizó Sánchez Albornoz en sus trabajos pioneros (1958) y también fue empleada por nosotros al iniciar las investigaciones sistemáticas en el PN Lago Puelo. Asimismo, otras fuentes que guiaron las recorridas fueron los informes de los guardaparques que, en el cumplimiento de sus tareas, identificaron sitios o materiales arqueológicos.

Esta estrategia de prospección dirigida fue complementada con recorridas a pie a lo largo de algunos de los senderos existentes dentro del Parque, en la margen Norte del lago Puelo y sobre el Cordón Derrumbe, en la margen Este (Figura 1). Se identificó la presencia de afloramientos rocosos hasta unos 50 metros a cada lado de los senderos, los que fueron inspeccionados en búsqueda de manifestaciones rupestres o de restos arqueológicos en superficie. Aun cuando no se observaron motivos rupestres, se tomaron fotos de todas las paredes para su posterior procesamiento con el software DStretch Image J (Decorrelation Stretch) (Harman 2008). Esto se hizo principalmente cuando personal de APN o los pobladores nos indicaron un sitio, cuando se observó algún trazo desvaído o se consideró que un afloramiento podría tener este tipo de vestigio. Esta decisión se basó en que los procesos de deterioro natural derivados de las condiciones climáticas del área producen el desvaído de las pinturas y la meteorización de pinturas y soportes (Caracotche et al. 2013, Podestá et al. 2000). 
Con el propósito de identificar ocupaciones que puedan vincularse con la navegación del lago, se realizaron prospecciones a pie en las playas consideradas aptas para un desembarco. Además, con el apoyo del personal del PNLP, se recorrieron todas las costas en una embarcación ${ }^{1}$. Se relevó la presencia de reparos y bloques, playas accesibles, cursos de agua y características de la topografía y de la vegetación. Se tomaron fotografías de las superficies rocosas para ser procesadas con el software mencionado anteriormente. En los puntos seleccionados para desembarcar se hizo una inspección visual hasta un máximo de 100 metros desde la costa, en busca de materiales arqueológicos en superficie. Todos los puntos de interés fueron fotografiados y georreferenciados.

\section{Pinturas rupestres, excavaciones y sondeos}

El relevamiento de los sitios con pinturas rupestres comprendió la caracterización de los soportes (ubicación, características, dimensiones, tipo de roca), la descripción de las representaciones teniendo en cuenta sus características morfo-tecnológicas y la documentación de los procesos de deterioro de los soportes y de los motivos pintados, que se hizo in situ y a partir de la aplicación del DStretch Image $J$ a las fotografías del soporte. Se realizaron sondeos y/o excavaciones en sitios seleccionados de acuerdo con su ubicación, las condiciones de reparo y el tipo de sedimento al pie de las pinturas. Los elegidos fueron Escuela 186 (ESC186), Alero de la Piedra Alta (APA) y Los Círculos (LC). En el primero se excavó 1,5 $\mathrm{m}^{2}$ y se alcanzó una profundidad máxima de 2,85 m. En APA la excavación está en curso y hasta el momento se intervino $1 \mathrm{~m}^{2}$ con una profundidad de $0,75 \mathrm{~m}$. En LC se realizó un sondeo de $0,25 \mathrm{~m}^{2}$ y 0,45 $\mathrm{m}$ de profundidad pero no se recuperaron materiales arqueológicos. En todos los casos, el sedimento extraído fue tamizado con zarandas de malla de $2 \mathrm{~mm}$. Los materiales recuperados se analizaron de acuerdo con los procedimientos establecidos en la bibliografía. En el caso de la cerámica, se relevó el estado de preservación, las superficies, pasta y decoración, a ojo desnudo y con lupa de mano sobre fractura fresca (Primera Convención Nacional de Antropología 1966; Rice 1987). Para los materiales líticos tallados se emplearon los criterios propuestos por Aschero $(1975,1983)$ y Aschero y Hocsman (2004). Se consideró el grupo tipológico, la materia prima, las dimensiones y el estado de las piezas (fracturas, alteraciones, pátinas, etc.). La observación de los artefactos fue hecha al nivel macroscópico, complementada con el uso de una lupa de mano de 10x. Para la caracterización tecno-morfológica de los litos modificados por uso (Aschero 1975, 1983) se consideraron las dimensiones, el estado de fragmentación y la materia prima. Las cuentas fueron analizadas a ojo desnudo, con lupa de mano de 20x y lupa binocular de 160x, siguiendo los criterios propuestos en Leonardt (2017). El análisis de los restos óseos faunísticos comprendió la determinación anatómica y taxonómica y su cuantificación (NISP y MNI),

\footnotetext{
${ }^{1}$ A diferencia del lago Nahuel Huapi, el lago Puelo no presenta islas cuya ocupación sería evidencia suficiente de navegación.
}

el relevamiento de las modificaciones de las superficies óseas, a ojo desnudo y con lupas de mano de 10x y 20x, la fragmentación y las evidencias de termoalteración (Lyman 1994; Mengoni Goñalons 1999).

\section{Resultados}

\section{Prospecciones}

Las prospecciones pedestres abarcaron aproximadamente $15 \mathrm{~km}$. En la parte Norte del PNLP se recorrieron $9 \mathrm{~km}$ del sendero que une el sector Los Hitos con la Planta Educativa y en el sector Este del Parque se relevaron $6 \mathrm{~km}$ del sendero que une El Desemboque con El Turbio, sobre el Cordón Derrumbe. En total se inspeccionaron 10 aleros/ bloques que por su ubicación y reparo podían haber sido utilizados. No se hallaron materiales en superficie ni manifestaciones rupestres visibles.

Las recorridas en embarcación cubrieron prácticamente todas las costas del lago y permitieron identificar nueve bloques/reparos y 10 playas practicables y de sustrato arenoso. No se hallaron pinturas rupestres ni materiales arqueológicos excepto los restos de dos "poblaciones", asentamientos de colonos que datan del siglo XX, ubicadas en el brazo occidental del lago. La primera se localiza en la costa Norte, entre el Destacamento de Gendarmería y Los Hitos, y comprende restos metálicos, de vidrio y maderas. La segunda está sobre la costa opuesta, en el Cerro Cuevas, cerca de la desembocadura del arroyo Melo y consiste en las ruinas de una construcción y de un corral. El empleo del programa DStretch Image J sobre las fotografías de las paredes de los reparos rocosos no reveló la existencia de motivos rupestres.

Las prospecciones dirigidas derivadas de los informes de guardaparques y/o de los relatos de los pobladores permitieron localizar cuatro sitios con pinturas rupestres, tres dentro del PNLP y uno en el PP. Los primeros se ubican sobre el Cordón Derrumbe y son los sitios APA, LC y LJ. La distancia lineal entre cada uno de ellos es de aproximadamente $500 \mathrm{~m}$ y se emplazan a diferentes alturas (340 msnm APA, 410 msnm LC y 600 msnm LJ) sobre una ladera cubierta de bosque. Todos están ubicados en afloramientos rocosos de grandes dimensiones rodeados de terreno plano circunscripto por bloques caídos. Presentan manifestaciones rupestres, un solo motivo en APA y LC y seis en LJ. El motivo de APA es difícil de percibir a simple vista porque está desvaído. Esta mala preservación se observa también en $L$, en donde hay tres motivos indeterminados debido al deterioro (Podestá et al. 2020). En APA y en LC se realizaron sondeos al pie de las pinturas pero sólo en APA se recuperó material arqueológico, que se presenta más adelante. En ocasión del primer reconocimiento del sitio LJ, efectuado en 1998 (Ballejos 1999), se constató la existencia de un pozo de saqueo debajo de las pinturas. Durante las tareas de remediación de este vandalismo se observó la presencia de materiales arqueológicos en estratigrafía. El sitio 
denominado Zanjón del Avellanal (ZJA) está ubicado en el PP, sobre el valle del río Turbio, aproximadamente a 14 $\mathrm{km}$ de la desembocadura del río en el lago Puelo. Está emplazado sobre un paredón de granito correspondiente a la primera estribación del faldeo (270 msnm) y presenta cinco motivos pintados. El talud está completamente cubierto por zarzamora/murra (Rubus ulmifolius).

\section{Manifestaciones rupestres}

Los resultados alcanzados en la documentación y caracterización de las pinturas rupestres del área son objeto de un trabajo específico, que desarrolla en detalle estos aspectos (Podestá et al. 2020). Brevemente, podemos señalar que los 10 sitios con pinturas rupestres identificados hasta el momento se localizan en el valle del río Turbio y en los faldeos del Cordón Derrumbe. La excepción es el sitio OLP, ubicado sobre un promontorio rocoso que se destaca de la línea de la costa del lago. Aunque es accesible por tierra (Sánchez Albornoz 1958), el soporte pintado está orientado hacia el lago, de allí que los motivos son mayormente visibles desde una embarcación.

La mayoría de los soportes utilizados para pintar son de granito y cubren diferentes tipologías, desde bloques pequeños, medianos y grandes hasta afloramientos rocosos más extensos. Destaca que, a pesar de la disponibilidad de soporte, las pinturas sólo cubren superficies de entre 2 y 3,60 $\mathrm{m}^{2}$. En algunos casos, los motivos se ubican en salientes naturales de los paredones, a más de un metro del suelo actual, lo que sugiere que se buscó darles mayor visibilidad a las manifestaciones (Podestá et al. 2020). El número total de motivos pintados considerando a los 10 sitios documentados ( $\mathrm{N}=54)$ es bajo en comparación con otros sectores de bosque (Podestá et al. 2020). En parte, su escasez podría deberse al desvanecimiento y exfoliación de las pinturas por efecto de las precipitaciones (Caracotche et al. 2013), la insolación y el accionar de colonias de microorganismos, procesos que se acentúan en este sector del bosque (Podestá et al. 2020).

Las pinturas corresponden a la TAGC (Gradin 1999) y a la MALB (Albornoz y Cúneo 2000) y están ejecutadas exclusivamente en rojo. Comprenden motivos geométricos simples (círculos, círculos concéntricos, cuadrados, líneas segmentadas o en zigzag, entre otros), complejos como las grecas, que son las más abundantes dentro del repertorio total de motivos, y representaciones figurativas de hachas en ocho y de placas grabadas o enmarcados con y sin diseños internos, además de arcos de hasta tres líneas, a la manera del arcoíris. Estos últimos pueden adscribirse a la MALB, identificada hacia el norte de nuestra área de estudio por Albornoz y Cúneo (2000). Se ha documentado una sola superposición en el sitio OLP, que es el que presenta la mayor cantidad de motivos (Podestá et al. 2020). En este mismo sitio, en la parte superior del panel pintado se relevó el grabado fuertemente patinado de una cruz latina, que podría representar la práctica colonial de contrarrestar la simbología indígena representada por las manifestaciones rupestres, con el propósito de extirpar idolatrías (Podestá et al. 2020), y por ende ser indicio de la circulación europea en el área (Figura 2).

Los sitios excavados

El sitio ESC186 está emplazado en un bloque de granito de aproximadamente $12 \mathrm{~m}$ de largo y presenta ocho motivos pintados, al pie de los cuales se realizó la excavación. De acuerdo con el relato de los pobladores, en las inmediaciones funcionó un destacamento de Gendarmería/Ejército entre 1950 y 1983, que utilizó el sitio como basural. Los primeros $40 \mathrm{~cm}$ de sedimento contienen desechos de esta ocupación. A partir del hallazgo de una lasca a dicha profundidad, se comenzó a remover el sedimento en niveles de $20 \mathrm{~cm}$ de espesor. El material recuperado es escaso (Tabla 1) y se dispone hasta aproximadamente 1,4 $\mathrm{m}$ de profundidad. Comprende un cepillo sobre nucleiforme de andesita hallado en superficie, 25 desechos de talla lítica, tres manos de molino de los cuales dos están enteras² (Figura 3, G), un fragmento de artefacto modificado por pulido no diferenciado y un pigmento. Los desechos de talla son muy pequeños (mediana de 18,5 mm) y son de obsidiana, riolita, andesita, dacita, calcedonia y sílice (Figura 3, J y K). De estas rocas, presumimos locales a las riolitas, andesitas y dacitas (Carballido Calatayud y Pereyra 2012). Se recuperaron 76 huesos, la mayor parte $(N=61)$ en el basural subactual. Excepto un fragmento asignado a artiodáctilo, el resto de los especímenes identificados anatómica y taxonómicamente corresponde a liebre (Lepus europaeus). Se detectó una estructura de combustión entre 0,7 y 0,85 $\mathrm{m}$ de profundidad desde la superficie, de la que se obtuvieron dos dataciones sobre carbón vegetal de $330 \pm 40$ y $500 \pm 40$ años AP (Bellelli et al. 2014). Su calibración (CALIB REV7.1.0 (c) M. Stuiver y P. J. Reimer 1986-2018) indica un rango entre 1401-1496 Cal AD (100\%, mediana Cal 1440 AD) y 1483-1663 Cal AD (100\%, mediana Cal 1566 AD). Diez centímetros por debajo del fogón afloran grandes rocas cuya forma y tamaño indican que se desprendieron de la pared-techo del sitio. Dado que los motivos están pintados sobre una "cicatriz" de derrumbe, es posible ubicar su ejecución con posterioridad a la caída de los bloques, muy probablemente dentro del rango cronológico de las dataciones.

El sitio APA está emplazado en medio del bosque, sobre un afloramiento rocoso cuyo frente se extiende por unos $50 \mathrm{~m}$, en un sector de aproximadamente 10 metros que posee una visera que genera un espacio protegido de unos $50 \mathrm{~m}^{2}$. Esta característica explica que, a nuestro arribo, el lugar presentaba un gran fogón reciente (1,2 x $1,2 \mathrm{~m})$, botellas plásticas, pavas, platos y una camada de hojas tipo "colchón". Según el relato del guardaparque Mario López, quien detectó el lugar en 2015, el alero funciona como eventual refugio desde hace varios años y el campamento fue desarmado por personal de Parques

\footnotetext{
2 Una de estas manos fue hallada por personal de la escuela, a unos metros del sitio arqueológico.
} 


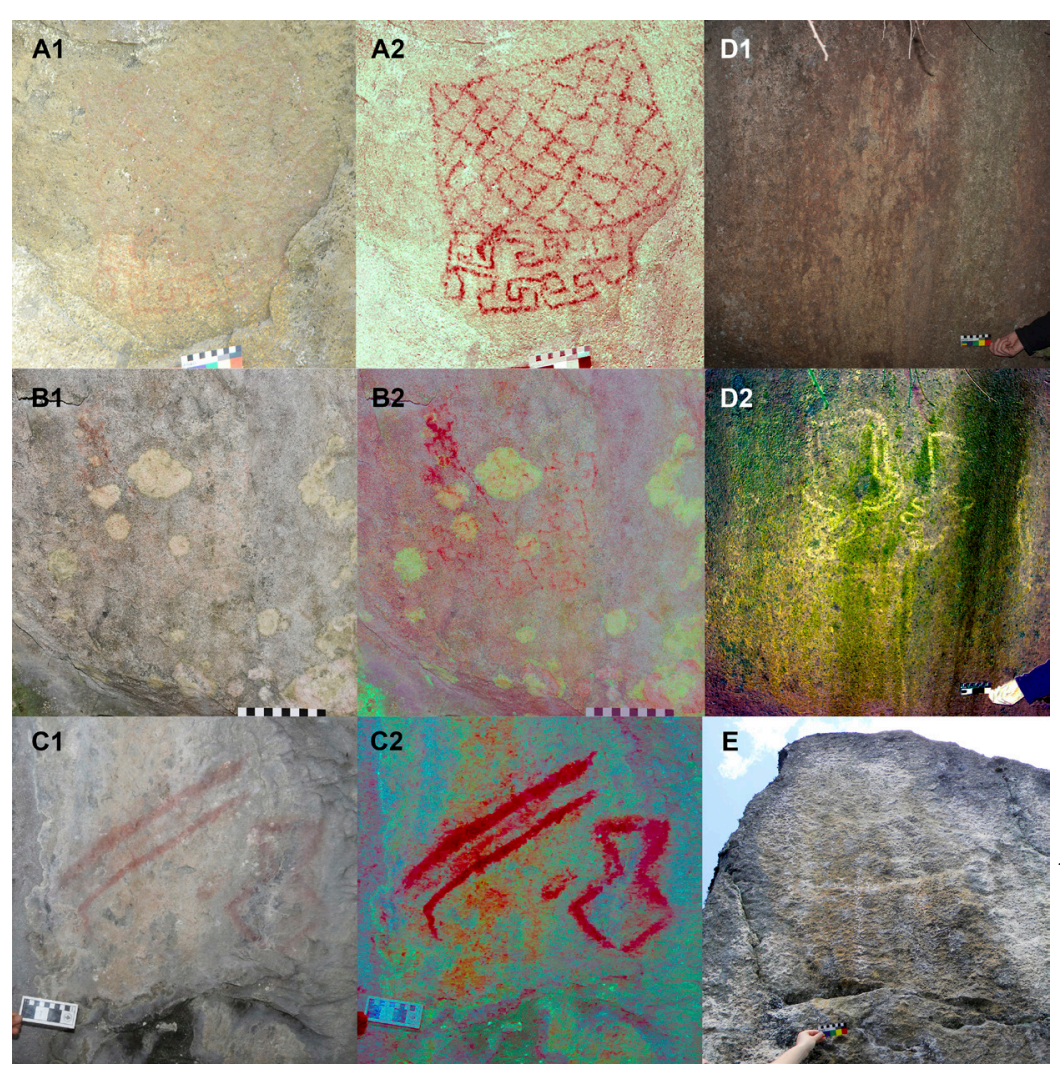

Figura 2. A: Sitio A orillas del lago Puelo. Enmarcado con diseños internos en rojo. A1 imagen al natural, $\mathrm{A} 2$ resaltada con DStretch (_yre). B: Sitio Alero de la Piedra Alta. Motivo geométrico complejo en rojo. B1 imagen al natural, B2 imagen resaltada con DStrecht ( $\_$re). C: Sitio La Jarra. Arco triple en rojo y figura romboidal (semejante a una jarra). C1 imagen al natural, C2 resaltada conDStretch (_Ire). D: Sitio Subida a la Vega del Turbio. Enmarcados de línea doble perimetral sin diseños internos, en rojo. D1 soporte natural donde no se perciben pinturas, D2 imagen resaltada con DStretch(_labi). E: Sitio A orillas del lago Puelo. Cruz cristiana grabada postcontacto. Escala: $10 \mathrm{~cm}$.

Figure 2. A: A orillas del lago Puelo site. Framed motif whith lines inside in red. A1 natural image, A2 enhanced with DStretch ( $(y r e)$. B: Alero de la Piedra Alta site. Geometrical motif in red. B1 natural image, B2 enhanced with DStretch (Ire). C: La Jarra site. Triple arch in red and rhomboidal figure (similar of a jar). C1 natural image, C2 enhanced with DStretch (_Ire). D: Subida a la Vega del Turbio site. Framed motives with double lines, D1 natural rock Surface without any visible motif, D2 enhanced with DStretch (Llabi). E: A orillas del lago Puelo site. Engraved Christian post-contact cross. Scale: $10 \mathrm{~cm}$.
Nacionales en varias oportunidades. En una de las paredes del sector protegido se registró una pintura rupestre de tipo geométrica compleja, casi imperceptible. Debajo se realizó la excavación, removiendo el sedimento en niveles de $10 \mathrm{~cm}$ de espesor. Como señalamos, la excavación del sitio continúa y hasta el momento constatamos que los materiales arqueológicos se registran hasta los $30 \mathrm{~cm}$ de profundidad, en una matriz sedimentaria limo arenosa, con clastos y bloques. Se identificaron dos estructuras de combustión y la datación de los carbones de una de ellas permite situar cronológicamente la ocupación de APA en 570 \pm 40 años AP (LP-3470). Su calibración (CALIB REV7.1.0 (C) M. Stuiver y P. J. Reimer 1986-2018) brinda un rango de entre 1322-1448 Cal AD (100\%, mediana 1412 Cal AD). Por debajo de los primeros $30 \mathrm{~cm}$ aparece una potente capa de ceniza volcánica de unos $40 \mathrm{~cm}$ de espesor, a la que sucede otra capa de sedimento limo arenoso.

Al igual que en la ESC186 los materiales recuperados son escasos (Tabla 1). Los instrumentos líticos tallados son un fragmento de punta de proyectil de sílice (Figura 3, I) y un fragmento de bifaz de calcedonia (Figura 3, H). Como en ESC186, hay 25 desechos de talla muy pequeños (mediana de $6 \mathrm{~mm}$ ), entre los que predomina la calcedonia, pero también hay obsidiana, sílice y basalto. Hasta el momento no se detectaron fuentes de materia prima en el área del PN Lago Puelo. No obstante, sobre la base de la estructura de recursos líticos de zonas cercanas, se presume que las tres primeras rocas serían alóctonas mientras que los basaltos podrían ser locales (Carballido Calatayud y Pereyra 2012). Se recuperaron dos tiestos cerámicos muy pequeños $(2,9$ y 3,3 cm²), de muy distinta morfología. Uno de ellos tiene siete milímetros de espesor y presenta la superficie externa marrón clara, alisada, con adherencias de material sedimentario y de sales; la superficie interna es gris oscura, alisada, también con aparentes adherencias de sedimento. El núcleo es marrón claro hacia los bordes virando al gris en el sector central, lo que indica una cocción oxidante algo incompleta. Las superficies de fractura poseen abrasión y están cubiertas de sedimento y adherencias de sales. La pasta es homogénea, uniforme, con pocas inclusiones muy pequeñas de mica y algunos granos de cuarzo. El otro tiesto es una pieza modelada, no tiene fracturas visibles y aparentemente se desprendió de una pieza mayor a la que estaba adosada. Se infiere que pudo haber sido una especie de asa o un elemento de adorno. Al no estar el núcleo expuesto no es posible describir la pasta. La superficie es marrón, alisada, con sectores más oscuros y con algo de brillo, producto del uso. También se recuperó un fragmento de molino y un artefacto sobre un fragmento de tibia de huemul, de morfología aguzada y terminación roma (Figura 3).

Entre los artefactos recuperados destacan 22 cuentas de pequeño tamaño, cuya intensa formatización dificulta la identificación del material sobre el que fueron elaboradas. Una primera caracterización a ojo desnudo y con lupa binocular determinó que una de las cuentas fue elaborada sobre un fragmento de mitílido -bivalvo marino ${ }^{3}$ - y el

\footnotetext{
3 La imposibilidad de determinar la especie de mitílido no permite precisar si proviene del litoral pacífico o atlántico.
} 


\begin{tabular}{|l|r|r|}
\hline & ESC186 & \multicolumn{1}{|c|}{ APA } \\
\hline Tipo & N & \multicolumn{1}{|c|}{$\mathrm{N}$} \\
\hline Instrumentos líticos tallados & 1 & 2 \\
\hline Desechos de talla lítica & 25 & 25 \\
\hline Molino & 0 & 1 \\
\hline Mano de molino & 3 & 0 \\
\hline Fragmento de artefacto pulido & 1 & 0 \\
\hline Tiestos & 0 & 2 \\
\hline Cuentas & 0 & 22 \\
\hline Artefacto óseo & 0 & 1 \\
\hline Pigmentos & 1 & 4 \\
\hline Restos óseos y dentarios & 76 & 314 \\
\hline Fragmentos de valva & 0 & 289 \\
\hline \multicolumn{1}{|c|}{ Total } & 107 & 660 \\
\hline
\end{tabular}

Tabla 1. Frecuencias de las evidencias recuperadas en los sitios Escuela 186 (ESC186) y Alero de la Piedra Alta (APA).

Table 1. Frequencies of evidences recovered at Escuela 186 (ESC186) and Alero de la Piedra Alta (APA) archaeological sites.

resto sobre roca. No obstante, el análisis de composición químico elemental con Fluorescencia de Rayos X, actualmente en curso, sugiere que todas las cuentas están elaboradas sobre valva ${ }^{4}$. Morfológicamente, todas tienen caras planas y perforación central, predominantemente bicónica. Excepto una que es subrectangular $(8,4 \times 5,9$ $\mathrm{mm})$, son de forma circular, con diámetro promedio de 4,28 mm (desvío estándar: 0,66 mm; mediana: 4,35 mm, Figura 3, A-E). El estado de preservación es bueno, ya que una sola cuenta registra evidencias de disolución. Otras ocho presentan indicios de estar quemadas. La homogeneidad morfológica y métrica del conjunto, recuperado en tan solo $1 \mathrm{~m}^{2}$, da sustento a la idea de que las cuentas formaron parte de un mismo artefacto. Tres cuentas con características similares fueron registradas en el valle del río Manso inferior, en niveles datados en los últimos 1200 años AP del sitio Población Anticura (Leonardt 2016) y en la margen noroccidental del lago Musters, en el sitio Delta Vulcana 1 con dataciones de ca. 1500 AP (Zubimendi y Moreno 2014).

Las evidencias orgánicas comprenden fragmentos de valva muy pequeños $(<5 \mathrm{~cm})$, cuyo análisis está en desarrollo, y restos faunísticos óseos y dentarios (Tabla 1). Entre los restos óseos identificados (NISP=233), el $93 \%$ corresponde a roedores, los que provienen de la parte basal de la estratigrafía y, por lo tanto, no están relacionados con la antigua ocupación del sitio. El resto del conjunto es informativo en términos de las especies representadas: huemul, pudú, chinchillón (Lagidium sp.), peces y aves paseriformes. Un hueso de ave tiene evidencias de corrosión gástrica, pudiendo formar parte de una egagrópila. Las trazas de acción antrópica se

\footnotetext{
${ }^{4}$ Estos análisis se desarrollan en el marco de la beca postdoctoral CONICET de Sabrina Leonardt, en colaboración con los Dres. Fernando Marte y Marcos Tascón, miembros del Instituto de Investigaciones sobre el Patrimonio Cultural de la Universidad de San Martín (IIPCTAREA, UNSAM).
}

limitan al artefacto sobre tibia de huemul (Figura 3, F) y a una huella de corte en un hueso indeterminado.

\section{Discusión}

La información generada nos permite comenzar a integrar el área de Lago Puelo con el resto de las zonas boscosas del SO de Río Negro y NO de Chubut. Si bien la investigación está en curso, la cronología disponible ubica a las ocupaciones en los momentos más tardíos de uso humano del bosque. Durante este lapso, el bosque del centro-norte de Patagonia está siendo usado de manera redundante y/o permanente. Por ejemplo, a $25 \mathrm{~km}$ lineales hacia el noreste de El Turbio, en el sitio Chacra de Lobos, hay restos humanos datados en 700 años AP que registran una señal isotópica que indica el consumo de proteínas animales del bosque y testimonian esa mayor permanencia (Fernández y Tessone 2014). Por el momento, en lago Puelo no se encontraron indicios de este tipo de uso pero consideramos que son necesarios más trabajos para contrastar adecuadamente esta posibilidad.

Al igual que en otras áreas de bosque que hemos investigado (Manso inferior y Cholila, Fernández et al. 2019b y Bellelli et al. 2020) en Puelo se obtuvo un fechado cuyo rango de calibración incluye a los tiempos históricos, que consideramos a partir de 1552-1567 AD (Fernández et al. 2019b). Más allá de la datación, no se recuperaron evidencias que puedan vincularse directamente con este lapso, tales como restos óseos de especies introducidas o materiales de origen europeo. Tampoco se identificaron motivos rupestres que puedan ser claramente relacionados con estos tiempos, a diferencia de lo que sucede en el Manso inferior (Podestá et al. 2020) o en el área del Nahuel Huapi (Albornoz y Hajduk 2009). No obstante, la ejecución de las pinturas podría haber tenido lugar durante parte de ese momento, y la cruz latina grabada en el sitio OLP sugiere algún tipo de presencia europea en el área (Podestá et al. 2020). Si bien no es posible precisar las razones o la cronología de esta presencia en Puelo, podría deberse a las expediciones esclavistas españolas del siglo XVII que partían desde Chiloé (Florez de León 1992) o a las misiones volantes que se realizaron desde Nahuel Huapi durante el tiempo que duraron las misiones en dicho lago (Urbina 2008).

Las características del arte rupestre son congruentes con esta cronología tardía, tanto por los motivos que pueden ser adscriptos a la TAGC como a la MALB. Esto permite vincular al área de Puelo con el resto de los sitios con manifestaciones rupestres trabajados por nosotros en el SO de Río Negro y NO de Chubut (Podestá et al. 2020). Mientras que esta adscripción estilística era conocida (Podestá et al. 2000), los nuevos estudios permiten identificar diferencias con los sitios de Cholila, Epuyén (Chubut), El Bolsón y Manso inferior (Río Negro). Estas comprenden la ausencia de sitios de grandes dimensiones y/o con gran cantidad de motivos, 

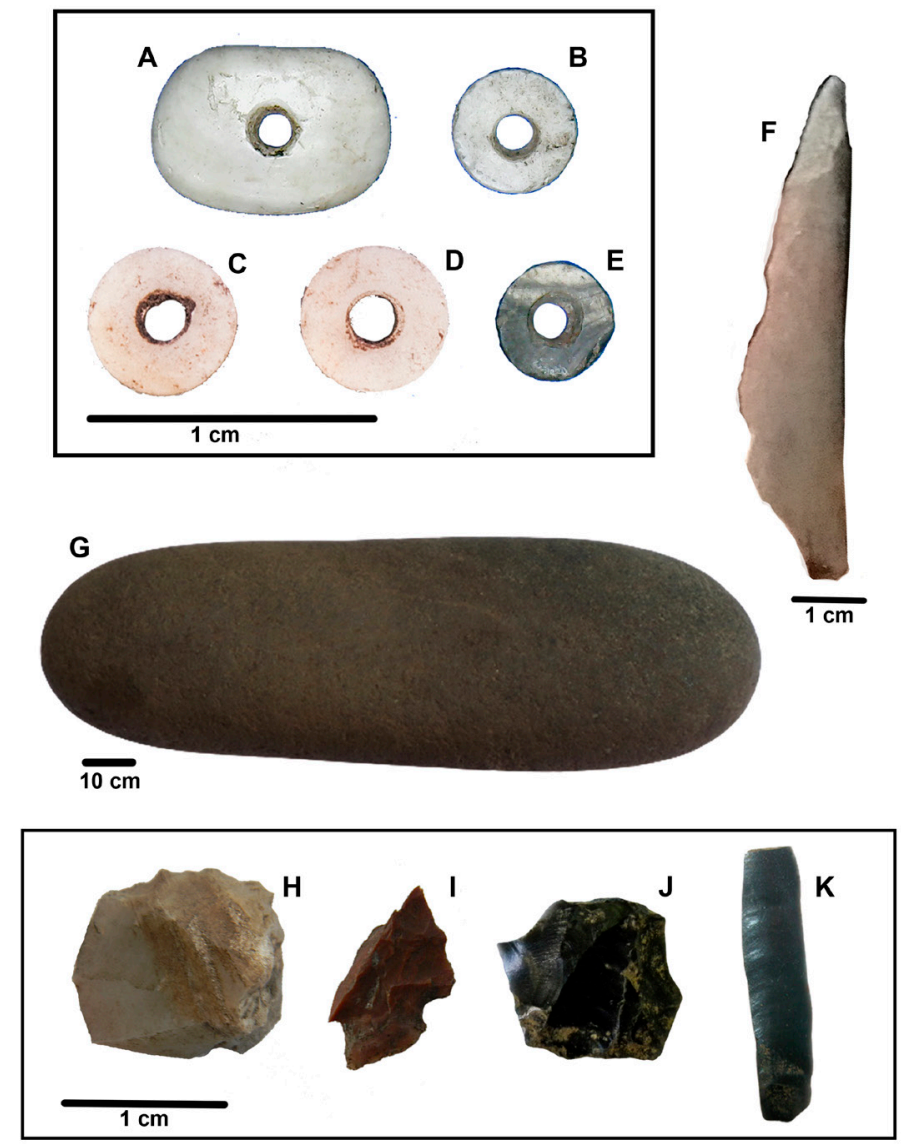

la escasez de superposiciones, la falta de motivos reciclados, de miniaturas y de diversidad cromática y la ausencia de la técnica de ejecución de trazo fino (Podestá et al. 2020). Algunas de estas características podrían explicarse por una menor circulación humana y/o una baja densidad poblacional, que habrían propiciado una menor redundancia de uso del espacio y de los sitios. Sin embargo, la falta de motivos miniaturizados o ejecutados con trazo fino estaría reflejando diferencias cronológicas. En el Manso inferior, la miniatura y el trazo fino se registran en sitios que poseen dataciones con fechados comprendidos en los tiempos históricos (Podestá et al. 2020). Aun en sitios sin cronología absoluta, la miniatura y el trazo fino aparecen en superposición, siempre arriba de los motivos preexistentes, tal como se observa en el sitio Gran Paredón de Azcona, de la cercana localidad de El Bolsón (Podestá et al. 2019). De todos modos, no podemos descartar por completo que tales diferencias pudieran ser también de índole idiosincrática.

En cuanto a la circulación dentro del PN Lago Puelo y el PP, la extensa red fluvio-lacustre del área y los antecedentes disponibles para otros lagos cordilleranos ubicados al Norte y al Sur del lago Puelo (ver resumen en Braicovich y Caracotche 2008) estimularon la búsqueda de indicadores de navegación, tales como restos de canoas o sitios sólo accesibles desde el agua. No obstante, la prospección sistemática de buena parte de las costas del lago practicables desde embarcaciones arrojó resultados negativos en términos arqueológicos.
Figura 3. Materiales arqueológicos recuperados durante la excavación de los sitios Escuela 186 (ESC186) y Alero de la Piedra Alta (APA). Referencias: A: cuenta de valva subrectangular. B-E: cuentas circulares (APA).. F: artefacto sobre tibia de huemul (APA). G: mano de molino (ESC186). $\mathrm{H}$ : fragmento de bifaz de calcedonia (APA). I: fragmento de punta de proyectil de sílice (APA). J: lasca de obsidiana (ESC186). K: lasca laminar de obsidiana (ESC186).

Figure 3. Archaeological evidences from Escuela 186 (ESC186) and Alero de la Piedra Alta (APA) sites. A-E: sub rectangular and discoidal shell beads (APA). F: bone tool on huemul tibiae (APA) G: mano. H: bifacial artifact, chalcedony (APA). I: projectile point fragment, silex (APA). J and K: obsidian lithic debitage (ESC186).
La única evidencia, que no es nueva, es el sitio OLP, que está orientado hacia el lago y que permite mantener la idea de la navegación como forma de circulación en el pasado. Además, cabe recordar que la expedición esclavista española documentada en el relato del capitán Juan Fernández accedió al lago Puelo navegando (Florez de León 1992). Esto sugiere que los guías nativos de los europeos conocían la factibilidad de navegarlo.

Así, la distribución espacial de los sitios documentados hasta el momento indica que la circulación terrestre fue tan importante como en otros sectores de nuestra área de investigación. Los sitios ubicados sobre el faldeo oeste del Cordón Derrumbe (APA, LC y L) están cercanos al sendero actual que conecta la zona de El Hoyo con el valle de El Turbio. A pesar de la relativa cercanía al sendero, no se disponen sobre él ni siguen su derrotero. Al contrario, se alejan de la senda moderna, que en este tramo discurre junto al lago: APA dista de ésta $180 \mathrm{~m}$, LC poco más de $500 \mathrm{~m}$ y $\mathrm{L}$ aproximadamente $1 \mathrm{~km}$. Otra característica notoria es que los tres sitios se ubican sobre una recta imaginaria aproximadamente a medio $\mathrm{km}$ de distancia entre sí, línea que sigue un sentido ascendente salvando un desnivel total de $260 \mathrm{~m}$ y que se orienta en dirección NO-SE (Figura 1). La ubicación de los sitios podría interpretarse como evidencia de una antigua vía de circulación, hipótesis a contrastar en el futuro. La idea de considerar a los sitios con pinturas como marcadores de rutas dentro del bosque ya ha sido postulada para otros contextos similares (ver discusión en Podestá et al. 2009). 
Otros tres sitios (SVT, ESC186 y PV) se ubican en un sector relativamente plano contiguo al río Turbio, en una cota de aproximadamente 240 msnm, unos $20 \mathrm{~m}$ por encima del cauce actual del río. El sitio PV, además, se emplaza cercano a un tributario del Turbio, que funciona como desagüe del lago Esperanza (Figura 1). De acuerdo con los pobladores, existen sitios con pinturas en las inmediaciones de dicho lago, zona desde la que es posible cruzar hacia Chile a través del Abra Esperanza. Hacia el Sur, sobre la cota de 400 msnm que corresponde a las primeras estribaciones del Cerro Cubridor, se ubica PQA, distante $1 \mathrm{~km}$ de la costa del río, por donde corre la senda actual. Ya dentro del PP se ubican los sitios RDT y ZJA, a 11 y 14 km del lago, respectivamente. Comparten la característica de estar emplazados cerca del río, sobre el primer afloramiento del faldeo y próximos al sendero actual. Al igual que PV, el sitio RDT está a unos metros de un curso tributario del Turbio, y en la zona donde comienza la senda actual que sube al Cerro Plataforma (1580 msnm). Hasta el momento, ZJA es el sitio más distante del lago aunque existen noticias de otros emplazados hacia las nacientes de río Turbio. Estos últimos, junto con ZJA, abren el interrogante sobre la naturaleza de la presencia humana en este sector del valle, que en este tramo se convierte en un virtual cul de sac, que desemboca en altas cumbres y glaciares. Como ya señalamos, en las fuentes históricas hay registros de que el lago Puelo fue alcanzado por la política esclavista de los españoles asentados al otro lado de la Cordillera de los Andes (Florez de León 1992). ¿Las "malocas" podrían haber forzado la ocupación de estos espacios alejados del lago, que fue la principal vía de acceso europeo? La excavación de alguno de estos sitios permitirá avanzar en la contrastación de esta hipótesis.

Por el momento, es posible plantear la concentración de nueve de los diez sitios conocidos en un sector de aproximadamente $20 \mathrm{~km}$ de extensión. Esta concentración no puede atribuirse al muestreo diferencial, ya que las caminatas y navegaciones buscaron cubrir distintos sectores dentro del PN y del PP. Asimismo, la información brindada por los pobladores señala a la zona que concentra a los sitios, a excepción de los datos sobre lago Esperanza y las nacientes del Turbio, que esperamos corroborar en las próximas campañas. Hasta el momento, en el patrón de distribución espacial observado destaca la ausencia de ocupación humana en los sectores de mayor precipitación del PN, ubicados hacia la frontera con Chile. Es posible que estos bosques más cerrados hayan sido aprovechados de manera diferente a las otras formaciones boscosas, quizá como lugares de aprovisionamiento de recursos vegetales específicos, así como espacios de tránsito siguiendo la vía lacustre-fluvial que conecta el área con el océano Pacífico. Asimismo, es posible que en los tiempos históricos la zona occidental del PNLP haya sido evitada para minimizar los contactos con las expediciones españolas.
Por último, a pesar de la escasa evidencia recuperada a escala de sitio, es posible recrear algunas de las relaciones que establecieron las antiguas poblaciones con su entorno. Entre otros aspectos, constatamos el aprovechamiento del huemul, evidenciado por el artefacto sobre una tibia de esta especie; el manejo de materias primas líticas no locales, como la obsidiana y posiblemente las rocas silíceas, así como las presumiblemente locales como basaltos y andesitas, y la relación con la costa marina, inferida a partir de una cuenta sobre valva de mitílido recuperada en APA. En relación con la tecnología cerámica, los dos tiestos recuperados en APA poseen cronología y características técnicas similares a las registradas en otros sitios de bosque de nuestra área de investigación (Bellelli 2020, Bellelli et al. 2020). La contemporaneidad y la ubicuidad de esta tecnología, así como su empleo para el procesamiento de los recursos animales (Chaile et al. 2018) sugieren que jugó un rol relevante en la explotación de los recursos del bosque en el tramo final del Holoceno.

\section{A modo de cierre}

Como señalamos, nuestro objetivo general es integrar la información arqueológica que venimos produciendo desde 2012 en el área del PN Lago Puelo con el resto de las áreas de bosque investigadas por nuestro equipo. La baja visibilidad, las malas condiciones de preservación, los desafíos logísticos y la cantidad de esfuerzo necesario para obtener datos no nos impidieron generar información cronológica, espacial, tecnológica, de subsistencia y de interacción con otras regiones, que permiten ampliar el conocimiento previo, basado principalmente en las manifestaciones rupestres. Como resultado, identificamos aspectos comunes con zonas vecinas así como algunas particularidades. Entre los primeros están el uso tardío del área, las características de las tecnologías cerámica y lítica, las relaciones con otros ambientes y el estilo de las manifestaciones rupestres. No obstante, estas últimas también poseen rasgos distintivos, algunos relacionados con su cronología y otros que podrían tener raíz idiosincrática. La concentración de sitios es otra diferencia. Estos resultados enriquecen la comprensión de la variabilidad de respuestas humanas en la ocupación del ambiente boscoso en esta latitud y estimulan la continuación de los trabajos en el área.

Buenos Aires, 11 de julio de 2020

\section{Agradecimientos}

Estamos profundamente agradecidos con los pobladores de El Turbio, Cholo Fernández, sus hijos Ariel y Adrián y sus esposas Bárbara y Nora; así como con la dirección de la Escuela 186, las/os maestras/os y todo el personal del establecimiento escolar. También disfrutamos de la hospitalidad de Beto Albornoz y su madre, la Sra. Ita (Barbarita) Sambueza, que nos dieron refugio en su casa y 
nos acompañaron en las recorridas. Asimismo, agradecemos a Alfredo Águila y a Fernando Saludes, por la colaboración brindada durante los trabajos de campo. El apoyo brindado por el personal de la Administración de Parques Nacionales fue y es clave para la realización de nuestros trabajos. Agradecemos al Intendente del PN Lago Puelo Sergio Rusak, a los guardaparques Marcos Ocampo, Lorena Loyza, Mario López, Marco Taraborrelli, Nicolás Ferrari y Mauricio Berardi, a los brigadistas Juan Vigueras, Juancho Fernández, Nazareno Vargas, Hernán Aravena, Marcelo Aguilera, Emiliano Leonardi, Matías Moraga, Andrés Pozas, Nicolás Vallejo y Julián Torres y al personal técnico Paula Werber y Nadia Zermatten. Carlos Masotta, antropólogo social que integra el equipo de investigación y director del Ubacyt 2016-2018 20020150100076BA (Universidad de Buenos Aires), participó de los trabajos de campo y contribuyó con sus ideas, debates y guisos al éxito de estos trabajos. Finalmente, agradecemos la atenta lectura y sugerencias de las/os dos evaluadoras/es anónimas/os. Las investigaciones fueron financiadas, además de la mencionada Universidad, por la ANPCyT (PICT 2012-0471), el Conicet (PIP 365/2014), y el Instituto Nacional de Antropología y Pensamiento Latinoamericano (Ministerio de Cultura de la Nación).

\section{Bibliografía}

Albornoz, A. M. \& Cúneo, E. (2000). Análisis comparativo de sitios con pictografías en ambientes lacustres boscosos de Patagonia Septentrional. En M. M. Podestá \& M. de Hoyos (Eds.), Arte en las Rocas. Arte Rupestre, menhires y piedras de colores en Argentina pp. 163-174. Buenos Aires SAA y AINA.

Albornoz, A. \& Hajduk, A. (2009). "Ladran Sancho I" Jinetes y caballos en el arte rupestre en la arqueología y la etnohistoria del área del Nahuel Huapi. Trabajo presentado a las XII Jornadas Interescuelas, Departamentos de Historia. Bariloche. Ms.

Aschero, C. (1975). Ensayo para una clasificación morfológica de artefactos líticos aplicada a estudios tipológicos comparativos. Informe presentado al CONICET. Manuscrito en archivo de CONICET.

Aschero, C. (1983). Ensayo para una clasificación morfológica de artefactos líticos. Apéndice $A$ y $B$. Manuscrito en archivo de la Cátedra de Ergología y Tecnología. Facultad de Filosofía y Letras, UBA.

Aschero, C. \& S. Hocsman (2004). Revisando cuestiones tipológicas en torno a la clasificación de artefactos bifaciales. En A. Acosta, D. Loponte y M. Ramos (comps.), Temas de Arqueología. Análisis Lítico: 7-25. Sociedad Argentina de Antropología, Instituto Nacional de Antropología y Pensamiento Latinoamericano y Departamento de Ciencias Sociales - Universidad Nacional de Luján, Luján.
Ballejos, L. (1999). Informe sobre el hallazgo de nuevas pinturas rupestres en el Parque Nacional Lago Puelo, Secc. Río Turbio. Informe presentado a la Administración de Parques Nacionales. Ms.

Bellelli, C. (2020). La cerámica acanalada de norpatagonia. Caracterización y cronología en el Valle del Río Manso (Río Negro). En V. Schuster y A. Pérez (eds.), Cerámica arqueológica de la Patagonia: 97-116. Fundación de Historia Natural Félix de Azara, Ciudad Autónoma de Buenos Aires.

Bellelli, C., Carballido Calatayud, M., Caracotche, S., Fernández, P. M. \& Podestá, M. (2014). Investigaciones arqueológicas en El Turbio (Parque Nacional Lago Puelo, Argentina). Póster presentado en las IX Jornadas de Arqueología de la Patagonia, Centro de Investigación en Ecosistemas de la Patagonia, Coyhaique, Chile. Disponible en http://www.saantropologia.com.ar/wp-content/ uploads/2015/04/Poster-Bellelli-et-al-IX-JAP-reduccion.pdf

Bellelli, C., M. Carballido Calatayud \& Ch. Stern (2018). Obsidianas en el bosque: determinación geoquímica de artefactos arqueológicos del S-O de Río Negro y $\mathrm{N}-\mathrm{O}$ de Chubut (Patagonia argentina). Chungara, Revista de Antropología Chilena 50(2): 201-216.

Bellelli, C., Fernández P. M. \& M. Carballido Calatayud (2020). Dataciones radiocarbónicas (AMS) de residuos orgánicos en tiestos del bosque norpatagónico. Revista Anuario de Arqueología. Volumen 12. En prensa.

Bellelli, C. V. Scheinsohn \& M. M. Podestá (2008). Arqueología de pasos cordilleranos: un caso de estudio en Patagonia norte durante el Holoceno tardío. Boletín del Museo Chileno de Arte Precolombino 13(2): 37-55.

Braicovich, R. \& Caracotche, S. (2008). Una biografía de las canoas monóxilas de la región andina norpatagónica. Perspectivas para su memoria y conservación. En III Jornadas de Historia de la Patagonia, San Carlos de Bariloche, 6-8 de noviembre de 2008, Sección E4 Antropología e Historia: interdisciplinariedad, convergencias disciplinares y estudios de caso en Patagonia. Publicado en formato CD Rom.

CALIB REV7.1.0 (c) M. Stuiver y P. J. Reimer 1986-2018. [WWW program] at http://calib.org

Caracotche, S., Podestá, M. \& Loyza, L. (2013). Pinturas rupestres del Parque Nacional Lago Puelo. Implicancias para su registro en bosques templados húmedos. Póster presentado al XVIII Congreso Nacional de Arqueología Argentina, Universidad Nacional de La Rioja, La Rioja, Argentina.

Carballido Calatayud, M. \& P. M. Fernández (2020). Hunting techniques along the rain shadow gradient in 
North- Central Patagonia, Argentina. En Ancient Hunting Strategies in Southern South America, J. B. Belardi, D. Bozzuto, P. M. Fernández, E. Moreno y G. Neme (Eds.). Springer AG. En Prensa.

Carballido Calatayud, M. \& F. X. Pereyra (2012). Determinación de la base regional de recursos líticos del área río Manso inferior-Foyel (Río Negro). Primeros resultados. Comechingonia. Revista de Arqueología 16: 287-296.

Faggi, A. M. (1994). Informe final correspondiente al relevamiento de las comunidades vegetales del Parque Nacional y Reserva Estricta Lago Puelo. Informe presentado a la Administración de Parques Nacionales. Ms.

Fernández, P. M. \& Carballido Calatayud, M. (2015) Armas y presas. Técnicas de caza en el interior del bosque patagónico. Relaciones de la Sociedad Argentina de Antropología XL (1): 279-301.

Fernández, P. M., Carballido Calatayud, M., Bellelli, C. T. \& Fernández, M. G. (2019b). Ocupaciones de tiempos históricos en el bosque del centro-norte de Patagonia argentina. En J. Gómez Otero, A. Svoboda \& A. Banegas (Eds.), Arqueología de la Patagonia: El pasado en las arenas pp. 105-116. Puerto Madryn Instituto de Diversidad y Evolución Austral.

Fernández, P. M., Carballido Calatayud, M., Bellelli, C. \& Podestá, M. (2013). Tiempo de cazadores. Cronología de las ocupaciones humanas en el valle del río Manso inferior (Río Negro). En: Zangrando, A. F.; Barberena, R.; Gil, A.; Neme, G.; Giardina, M.; Luna, L.; Otaola, C.; Paulides, S.; Salgán, L. \& Tivoli, A. (eds.): Tendencias teóricometodológicas y casos de estudio en la arqueología de Patagonia, pp. 167-175. Museo de Historia Natural de San Rafael, Sociedad Argentina de Antropología e Instituto Nacional de Antropología y Pensamiento Latinoamericano. Buenos Aires.

Fernández, P. M., Carballido Calatayud, M., Bellelli, C. T., Tchilinguirián P., Leonardt, S. \& Fernández, M. G. (2019a). Nuevos datos sobre el poblamiento inicial del bosque del centro-norte de Patagonia, Argentina. Latin American Antiquity 30(2): 300-317.

Fernández, P. M. \& M. G. Fernández (2019). Zooarqueología de tiempos históricos en el bosque andino de la Patagonia argentina. Continuidades y cambios en el valle del Manso inferior. Archaeofauna. International Journal of Archaeozoology 28: 51-60.

Fernández, P. M. \& Tessone, A. (2014). Modos de ocupación del bosque patagónico de la vertiente oriental de los andes: aportes desde la ecología isotópica. Revista Chilena de Antropología 30: 83-89.
Florez de León, D. (1992). Florez de León, Diego "Memorial". Publicación del Museo de la Patagonia F. P. Moreno, Bariloche.

Gradin, C. (1999). Sobre las tendencias estilísticas del arte rupestre de Patagonia argentina. En Segundas Jornadas de Investigación en Arqueología y Etnohistoria del Centro-Oeste del País, pp. 85-99. Río Cuarto Universidad de Río Cuarto.

Hajduk, A., A. Albornoz \& M. Lezcano (2006). Levels with Extinct Fauna in the Forest Rockshelter El Trébol (Northwest Patagonia, Argentina). Current Research in the Pleistocene 23: 55-57.

Hajduk, A., A. M. Albornoz \& M. Lezcano (2011). Espacio, cultura y tiempo: el corredor bioceánico norpatagónico desde la perspectiva arqueológica. En P. Navarro Floria y W. Delrio (eds.), Cultura y espacio. AraucaníaNorpatagonia: 262-292. San Carlos de Bariloche, IIDyPCa, Universidad Nacional de Río Negro.

Hajduk, A., F. Scartascini, F. E. Vargas \& M. Lezcano (2018). Arqueología de la Isla Victoria, Parque Nacional Nahuel Huapi, Patagonia Argentina: actualización y perspectivas futuras. Intersecciones en Antropología 19: $37-48$.

Harman, J. (2008 [2005]). Using Decorrelation Stretch to enhance rock art images. Recuperado de: http://www. dstretch.com

Leonardt, S. (2016). Variabilidad temporal en la producción de artefactos de adorno personal en Patagonia continental: análisis a partir del sitio Población Anticura (provincia de Río Negro, Argentina). Magallania 44 (1): 229-247.

Leonardt, S. (2017). Producción y distribución de cuentas de valva en el Holoceno tardío de Patagonia continental argentina. Tesis de doctorado inédita. Facultad de Filosofía y Letras, Universidad de Buenos Aires.

Lezcano, M. J., A. Hajduk \& A. Albornoz (2010). El menú a la carta en el bosque ¿entrada o plato fuerte?: una perspectiva comparada desde la zooarqueología del sitio El Trébol (lago Nahuel Huapi, Pcia. de Río Negro). En Zooarqueología a principios del siglo XXI: aportes teóricos, metodológicos y casos de estudio, editado por M. A. Gutiérrez, M. De Nigris, P. M. Fernández, M. Giardina, A. F. Gil, A. Izeta, G. Neme y H. D. Yacobaccio, pp. 243-257. Ediciones del Espinillo, Buenos Aires.

López, M. (2015). Base de Datos del Patrimonio Cultural de la Administración de Parques Nacionales. Sistema de Información de Biodiversidad, Administración de Parques Nacionales. 
Lyman, R. L. (1994). Vertebrate Taphonomy. Cambridge University Press, Cambridge.

Masotta, C., Fernández, P. M., Bellelli, C. \& Carballido Calatayud, M. (2016). La lección de la clepsidra. Interrogantes sobre las pinturas rupestres y el tiempo en el valle del río Turbio (Parque Nacional Lago Puelo - provincia de Chubut). En F. Oliva, A. Rocchietti \& F. Solomita Banfi (Eds.), Imágenes Rupestres: lugares y regiones pp. 547-555). Rosario: Facultad de Humanidades y Artes, Universidad Nacional de Rosario.

Mengoni Goñalons, G. L. (1999). Cazadores de guanacos de la estepa patagónica. Buenos Aires, Colección Tesis Doctorales, Sociedad Argentina de Antropología.

Molinari, R. (1991). Informe de la Comisión al Parque Nacional Lago Puelo. Relevamiento de las pinturas rupestres. APN. MS

Ocampo M. \& H. Giaccino (2005). Base de Datos del Patrimonio Cultural de la Administración de Parques Nacionales. Sistema de Información de Biodiversidad, Administración de Parques Nacionales.

Pérez, A. (2010). Arqueología del bosque. El registro arqueológico del interior y borde de bosque de Norpatagonia. En Actas del XVII Congreso Nacional de Arqueología Chilena 2, pp. 1515-1528. Sociedad Chilena de Arqueología-Universidad Austral de Chile-Kultrún, Valdivia.

Pérez, A. \& V. Reyes (2009). Técnica improntas de hojas. Algunas reflexiones acerca de su novedoso registro en la vertiente oriental de la Cordillera de los Andes. Magallania 37(1): 113-132.

Pérez, A., A. Tessone \& J. L. Lanata (2019). Paleodietas en restos humanos del bosque meridional de Neuquén, Patagonia Argentina. Magallania 47(2): 183-191.

Podestá, M. M., Albornoz, A., Vasini, A. \& Tropea, E. (2009). El sitio Peumayén 2 en el contexto del arte rupestre del bosque andino-patagónico. Comechingonia Virtual III (2): 117-153.

Podestá, M. M., Bellelli, C., Fernández, P., Carballido M. \& Paniquelli, M. (2000). Arte rupestre de la Comarca Andina del Paralelo 42: Un caso de análisis regional para el manejo de recursos culturales. En M. M. Podestá \& M. de Hoyos (Eds.), Arte en las rocas. Arte rupestre, menhires y piedras de colores en la Argentina pp. 175-201. Buenos
Aires Sociedad Argentina de Antropología.

Podestá, M. M., Caracotche, S., Bellelli, C. \& Forlano, A. (2020). Evidencias del arte rupestre tardío en el bosque patagónico, las pinturas del Lago Puelo (Chubut, Argentina). Cuadernos del INAPL. Series Especiales. En prensa.

Podestá, M. M., Romero Villanueva, G., Bellelli, C., Forlano, A. \& Caracotche, S. (2019). Gran Paredón de Azcona (Provincia de Río Negro, Patagonia): puesta al día en la documentación de sus pinturas rupestres (1955-2015). Revista Sociedades de Paisajes Áridos y Semi-Áridos XII (2):177-197.

Primera Convención Nacional de Antropología (1966). Publicaciones 1 (26), Primera parte. Facultad de Filosofía y Humanidades, Instituto de Antropología, Córdoba.

Rice, P. M. (1987). Pottery analysis. A sourcebook. University of Chicago Press. Chicago y Londres.

Sánchez Albornoz, N. (1958). Pictografías del Valle del Bolsón y Lago Puelo. Acta Praehistorica II: 146-175.

Scheinsohn, V. (2004). "En el país de los ciegos, el tuerto es rey". Visibilidad arqueológica y paisaje en la localidad Cholila. En M. T. Civalero, P. M. Fernández y A. G. Guráieb (comps.), Contra Viento y Marea. Arqueología de Patagonia 581-590. Buenos Aires, Instituto Nacional de Antropología y Pensamiento Latinoamericano y Sociedad Argentina de Antropología.

Silveira, M., L. López \& V. Aldazabal (2013). El uso del espacio durante el Holoceno tardío -últimos 3500 añosen bosque andino de Patagonia septentrional, lago Traful, sudoeste de la provincia de Neuquén. Anuario de Arqueología, 5: 85-101.

Silveira, M., L. López \& V. Aldazabal (2014). Investigaciones arqueológicas en el alero Las Mellizas, bosque andino de Patagonia septentrional, sudoeste de la provincia de Neuquén. Comechingonia Virtual VIII (2): 157-190.

Urbina, M. X. (2008). La frustrada misión estratégica de Nahuelhuapi, un punto en la inmensidad de la Patagonia. Magallania 36(1): 5-30.

Zubimendi, M. A. y Moreno, J. E. (2014). La presencia de artefactos arqueomalacológicos en la localidad arqueológica Delta del arroyo Vulcana (lago Musters, provincia del Chubut). Intersecciones en Antropología 15: 71-87. 